\title{
Mejora de la motivación en estudiantes inmigrantes latinoamericanos mediante un programa de mindfulness: un estudio aleatorizado
}

\section{Improving motivation in Latin American immigrants through a mindfulness- based program: a randomized study}

\author{
Clemente Franco \\ Universidad de Almería, España \\ Encarnación Soriano \\ Universidad de Almería, España \\ Alberto Amutio \\ Universidad del País Vasco (UPV/EHU), España \\ Universidad Andres Bello, Chile \\ Israel Mañas \\ Universidad de Almería, España \\ Universitat Oberta de Catalunya, España
}

Rec (23 de Noviembre de 2019) Acept (24 de Enero de 2020)

\footnotetext{
Correspondencia: Universidad del País Vasco (UPV/EHU), Alberto Amutio Kareaga, Ph.D. (buzón 57), Facultad de Relaciones Laborales y Trabajo Social,Bº Sarriena, s/n. Campus de Leioa - 48012, Bizkaia (SPAIN). e-mail: alberto.amutio@ehu.eus Wordcount: 7439 


\title{
Resumen
}

En este estudio experimental se presentan los resultados de un programa psicoeducativo de mindfulness sobre la motivación del logro y motivación para el aprendizaje aplicado a una muestra de estudiantes inmigrantes de origen latinoamericano que viven en el sureste español. La muestra se compone de 50 estudiantes, de los cuales 25 forman el grupo experimental y 25 el grupo de control. El programa de intervención se llevó a cabo durante 10 semanas. Los resultados muestran diferencias estadísticamente significativas entre los grupos en la variable motivación de logro y en tres de las seis dimensiones de la variable motivación para el aprendizaje (autoeficacia, control y ansiedad). El estudio confirma la eficacia del programa de mindfulness para mejorar los niveles de motivación del logro y la motivación para el aprendizaje en los jóvenes latinoamericanos procedentes de la inmigración. Se destaca la conveniencia de la utilización de estos programas dentro del currículum educativo.

Palabras clave: Estudiantes latinoamericanos; inmigrantes; mindfulness, motivación de logro; motivación para el aprendizaje.

\begin{abstract}
In this experimental study, we present the results of a mindfulness psycho-educational program on achievement motivation and motivation for learning applied to a sample of immigrant students of Latin American origin living in southeastern Spain. The sample consists of 50 students, of which 25 form the experimental group and 25 the control group. The intervention program was carried out for 10 weeks. The results show statistically significant differences between both groups in motivation for achievement and in three of the six dimensions of motivation for learning (self-efficacy, control and anxiety). The study confirms the effectiveness of the mindfulness program to improve the levels of achievement motivation and motivation for learning in Latin American youth immigrants. The convenience of using these programs within the educational curriculum is highlighted.

Keywords: Latin American students; immigrants; mindfulness; achievement motivation; motivation for learning.
\end{abstract}

\section{Introducción}

El estudio de los factores de orden motivacional y afectivo que inciden sobre el aprendizaje y al rendimiento académico son tópicos de investigación en alza que están atrayendo el interés de numerosas investigaciones en los últimos años (Franco, Beja, Candeias, \& Santos, 2017; Karimi \& Sotoodeh, 2019; Rodríguez, Núñez, Valle, Freire, Ferradas, \& Rodríguez-Llorente, 2019). Corno \& Snow (1986) establecían la existencia de dos variables relevantes en el aprendizaje escolar, como son la personalidad (e.g., ansiedad, autoconcepto) y la motivación académica, de tal modo, que la implicación activa en el proceso de aprendizaje aumenta cuando el estudiante se siente autocompetente, confía en sus propias capacidades y mantiene altas expectativas de autoeficacia. También aumenta cuando valora las tareas y cuando se siente responsable de los objetivos de aprendizaje, dando lugar con ello a un incremento y mejora en su rendimiento académico (González-Pienda, Núñez, González-Pumariega, \& García, 1997; Gutiérrez \& López, 2012).

Diversos estudios e investigaciones, muestran que cuanto mayor es el autoconcepto positivo del estudiante, mejores son las condiciones motivacionales que presenta para el estudio (menor ansiedad, mayor concentración y mejores aptitudes hacia el trabajo escolar), y mayores las estrategias de apoyo al estudio y estrategias de aprendizaje profundo que emplea. De este modo, el autoconcepto positivo favorece la utilización por parte del estudiante de más estrategias cognitivas de aprendizaje cuyo uso facilita un procesamiento más profundo y elaborado de la información (Barca-Lozano, Almeida, Porto-Rioboo, Peralbo-Uzquiano, \& Brenlla-Blanco, 2012; López-González, Amutio, Oriol, \& Bisquerra, 2016).

Los modelos de aprendizaje autorregulado permiten identificar otras variables (a parte de las cognitivas), que el estudiante pone en juego en su proceso de aprendizaje, como son las variables de carácter emocional (Amutio, Franco, Gázquez, \& Mañas, 2015; Covington, 2000), comportamental (Boekaerts \& Pekrum, 2005), y de tipo volitivo (Etxebarria, Ortiz, Apodaca, Pascual, \& Conejero, 2015; Maquilón \& Hernández, 2011), todas 
ellas, variables relacionadas con el bienestar y el equilibro personal del estudiante. Actualmente un factor clave en la motivación y el rendimiento es el de grit, que se define como "perseverancia y pasión por los objetivos a largo plazo" (Duckworth, Peterson, Mathews, \& Kelly, 2007, p. 1087). Grit se relaciona estrechamente con el autocontrol, la satisfacción con la escuela, y el rendimiento académico (Lam \& Zhou, 2019; Oriol, Miranda, Oyanedel, \& Torres, 2017). Por su parte, la ansiedad, la falta de habilidades de regulación emocional, así como niveles bajos de competencia y de comprensión emocional, están relacionados con niveles bajos en el desempeño académico (Carbonero, 1999; Oriol, Amutio, Mendoza, Da Costa, \& Miranda, 2016; Franco et al., 2016). De igual manera, las emociones negativas respecto al estudio y la realización de exámenes influyen negativamente en el rendimiento académico y en la motivación debido a la focalización del estudiante en pensamientos negativos respecto a sus capacidades más que en la tarea (Boeckaerts \& Pekrun, 2015; Carbonero, 1999).

Diversos estudios ponen de relieve la relación y utilidad de las técnicas basadas en la atención plena (mindfulness) sobre los procesos de regulación emocional. Kabat-Zinn (2003) describió mindfulness como la capacidad de llevar la atención a las experiencias que se están experimentando en el momento presente, de un modo particular, aceptándolas y sin juzgar. De forma más general, mindfulness ha sido descrito como una clase de conciencia centrada en el presente, no elaborativa ni condenatoria, en la cual cada pensamiento, sentimiento o sensación que surge en el campo atencional es reconocido y aceptado tal y como es.

La psicología contemporánea emplea las técnicas basadas en mindfulness para incrementar la consciencia y responder de manera adaptativa a los procesos mentales que contribuyen al desarrollo del malestar emocional y a comportamientos desadaptativos. En este sentido, mindfulness interfiere en la regulación de sistemas de respuestas defensivas disminuyéndolas y contribuye al desarrollo de conductas más adaptativas, incluyendo un incremento de la conciencia de múltiples perspectivas en la resolución de problemas (Amutio et al., 2015; Langer, 2000). El estudio de Ortner, Kilner, y Zelazo (2007) mostró que mindfulness puede reducir la interferencia emocional sobre tareas de tipo cognitivas atenuando la reactividad emocional prolongada ante los estímulos emocionales. En la misma línea, Evans, Baer, y Segerstrom (2009) hallaron en una muestra de estudiantes de psicología, que mindfulness, y particularmente las facetas de no reaccionar y no condenar, incrementa la persistencia sobre tareas de laboratorio con ciertos grados de dificultad a través de procesos de auto-regulación. Adicionalmente, Feltman, Robinson, y Ode (2009) encontraron en una muestra de estudiantes universitarios, que a través de mecanismos de auto-regulación, mindfulness juega un papel importante como moderador de variables relacionadas con el neuroticismo y la ansiedad, que son predictoras de respuestas emocionales negativas. Por su parte, De la Fuente, Franco, y Mañas (2010) hallaron efectos positivos en el estado emocional en una muestra de estudiantes universitarios tras la aplicación de un programa de intervención basado en mindfulness. Más específicamente, el estudió mostró que el programa producía una reducción significativa en el cansancio emocional y un incremento del engagement académico, que es un estilo de motivación favorable y duradero hacia los aspectos relacionados con el estudio. Los resultados positivos se mantuvieron en las medidas de seguimiento trascurridos seis meses. Estos resultados se hayan en consonancia con otros estudios en diversas poblaciones (Klingbeil \& Renshaw, 2018; Krick \& Felfe, 2019; Blanco-Donoso, García-Rubio, Moreno-Jiménez, De la Pinta, Moraleda-Aldea, \& Garrosa, 2017).

Diversas investigaciones muestran cómo la práctica continuada de mindfulness produce numerosos efectos beneficiosos sobre el organismo y sobre la actividad psicológica de las personas que lo practican. Su efectividad ha sido demostrada en multitud de problemas médicos y psicológicos, así como también en la mejora de la calidad de vida de las personas, siendo sus aplicaciones muy numerosas y diversas (véanse exhaustivas revisiones en Dunning et al., 2019; Goldberg et al., 2018; Roche, Kroska, \& Denburg, 2019; Weinstein, Brown, \& Ryan, 2009; Sevilla, Santesteban, Pryor, McGorry, \& Álvarez, 2018; Solhaug et al., 2019). Uno de los ámbitos de actuación donde mindfulness también ha demostrado ser efectivo es el ámbito educativo en diferentes variables, y lo ha hecho de múltiples formas (Cheng, 2016; Franco, 2009a; Langer, Ulloa, Cangas \& Rojas, \& Krause, 2015; León, 2008; López-González et al., 2016; López-González, Amutio, \& Herrero, 2018; Sapthiang, Van Gordon, \& Shonin, 2019). Sin embargo, aún resulta necesario seguir realizando investigaciones ya que hay estudios en donde no se han obtenido diferencias estadísticamente significativas entre el grupo de intervención y el control (Oblitas, Soto, Anicama, \& Arana, 2019). 


\section{El contexto de la investigación}

En los últimos años el proceso migratorio ha experimentado grandes transformaciones en países como Chile (Caqueo-Urízar, Flores, Irarrázaval, Loo, Páez, \& Sepúlveda 2019) y España. En el caso concreto de la sociedad española, la situación económica de finales de los años noventa motivó un boom de atracción migratoria entre 1998-2008 (Franco, Soriano \& Justo, 2010; Reher, Requena, \& Sanz, 2011) caracterizado por la llegada masiva de personas procedentes del norte de África, Europa del Este y Latinoamérica. En este periodo la proporción de inmigrantes en España se vio quintuplicada (Arango, 2004; Soriano, 2012). El carácter económico de las migraciones dio lugar a un perfil migratorio de población joven y familias con hijos. Actualmente según el Ministerio de Educación y Formación Profesional (2019) el porcentaje de niños y adolescentes inmigrantes ha aumentado. Así, en el curso 2017-18 ascendió a 751.404 alumnos en las enseñanzas no universitarias, de los cuales el $34.06 \%$ proceden de Europa, de África el $30.58 \%$ y de América Central y Sur el $23.59 \%$. Por países destacan el alumnado procedente de Marruecos (181.833); Rumanía (105.603), Ecuador (27.976), China (41.364) y Colombia (22.265).

Los datos del informe PISA han mostrado de forma sistemática una brecha de rendimiento entre estudiantes de origen inmigrante y "autóctonos" (OECD, 2015). El estudiante inmigrante tiene que superar a menudo múltiples barreras de forma simultánea para lograr el éxito en la escuela: la barrera lingüística, su propia condición de persona inmigrada, un contexto desfavorecido, y los obstáculos habituales en el aprendizaje comunes a otros compañeros de clase. La capacidad de los estudiantes para superar estas dificultades y ser resilientes se refleja no solo en su habilidad para alcanzar niveles básicos de competencia académica, sino también en su sentido de pertenencia a la escuela, su satisfacción con la vida, su nivel de trabajo escolar y su motivación para el logro. En España los hijos de familias inmigradas según el informe PISA tenían un sentido de pertenencia débil con la escuela, eran más propensos a sufrir problemas emocionales y manifestaban baja satisfacción con la vida (OECD, 2018). Aunque los estudiantes con los que llevamos a cabo la intervención no presentan problemas lingüísticos significativos, puesto que todos ellos hablan una variedad del español, si participan de las otras barreras, entre ellas, el hecho de que sus padres tienen recursos económicos limitados, y viven en barrios de la periferia, por lo que coinciden un gran número de ellos en un mismo centro educativo. Por ello, y teniendo en cuenta la influencia del contexto cultural sobre el bienestar en los seres humanos (Gutiérrez-Carmona \& Urzúa, 2019), como educadores y psicólogos podemos ayudar a disminuir el choque cultural, el estrés de aculturación y favorecer su integración escolar y social logrando que mejoren el rendimiento escolar, incrementando para ello su motivación para el aprendizaje y el logro (Martínez-Taboada, Mera, Amutio, Castañeda, Felt \& Nicolae 2017; Urzua, Basabe, Pizarro, \& Ferrer, 2017; Soriano, 2005).

\section{Objetivo e hipótesis}

El objetivo de la presente investigación fue examinar los efectos de un programa psicoeducativo basado en mindfulness (Meditación-Fluir) aplicado a un grupo de adolescentes inmigrantes de origen latinoamericano residentes en el sudeste español, sobre sus niveles de motivación de logro y de motivación para el aprendizaje. Se hipotetizó que los sujetos expuestos al tratamiento obtendrían un incremento en la variable motivación de logro y una mejora en las dimensiones de la variable motivación para el aprendizaje en comparación con los sujetos del grupo control.

\section{Método}

\section{Participantes}

En el presente estudio participaron un total de 50 estudiantes inmigrantes de origen latinoamericano residentes en el sudeste español que cursaban primer curso de Bachillerato. El $52 \%$ de los participantes eran chicas y el $48 \%$ chicos, cuyas edades oscilaban entre los 16 y los 18 años de edad (Media $=16.45$; Desviación típica $=0.78$ ). El grupo control estuvo formado por 25 sujetos ( $56 \%$ chicas y $44 \%$ chicos), y los otros 25 formaron parte del grupo experimental ( $48 \%$ chicas y $52 \%$ chicos). 
Con el fin de analizar los efectos del programa Meditación-Fluir (variable independiente) sobre la motivación de logro y las distintas dimensiones de la variable motivación para el aprendizaje (variables dependientes), se utilizó un diseño experimental de comparación de grupos con medidas pre-test/post-test, con un grupo experimental y un grupo control.

\section{Instrumentos}

Para la evaluación de las variables del estudio, se aplicaron los siguientes cuestionarios:

- Escala Atribucional de Motivación de Logro (EAML) (Manassero \& Vázquez, 1998). Compuesta por 22 ítems basados en el diferencial semántico de Osgood sobre una escala de 1-9 puntos, de tal manera que una mayor puntuación refleja una motivación más favorable para el éxito escolar. Esta prueba muestra un índice de fiabilidad (alfa de Cronbach) de .862 para la muestra estudiada.

- Cuestionario de Estrategias y Motivación para el Aprendizaje (MSLQ) (Pintrich, Smith, García, \& Mc Keachi, 1991). Se aplicaron únicamente los 31 ítems que evalúan la variable motivación para el aprendizaje mediante una escala tipo Likert de 7 puntos, que a su vez está compuesta por 6 dimensiones:

- Motivación intrínseca (4 ítems): que evalúa el grado en que el alumnado realiza las actividades por el interés que proporcionan en sí mismas dichas actividades.

- Motivación extrínseca (4 ítems): hace referencia a en qué medida el alumnado realiza las actividades no por la actividad en sí, sino para conseguir otras metas o fines.

- Valoración de la tarea (6 ítems): se refiere a en qué medida el alumnado considera que las actividades académicas son útiles e interesantes.

- Creencias de autoeficacia (8 ítems): refleja el grado en que el alumnado se considera capaz para realizar de forma eficaz las tareas académicas.

- Creencias de control (4 ítems): evalúa las creencias del alumnado sobre su grado de control respecto a su aprendizaje y rendimiento académico.

- Ansiedad (5 ítems): mide el grado de ansiedad que las situaciones de aprendizaje y las actividades académicas generan en el alumnado.

El índice de fiabilidad de la prueba arrojó un alfa de Cronbach de .793 para la muestra estudiada.

\section{Procedimiento}

En primer lugar, se procedió a la selección de los sujetos participantes en la investigación. Para ello se contactó con los directores de los diferentes centros educativos participantes en el estudio, con el fin de ofertar una actividad extraescolar titulada "Aprendizaje y práctica del mindfulness en el contexto escolar", dirigida al alumnado de origen latinoamericano que se encontraban cursando primer curso de Bachillerato. A la actividad se inscribieron un total de 54 estudiantes, de los cuales 50 pasaron a formar parte de la investigación, ya que no se tuvieron en cuenta para los resultados del estudio aquellos estudiantes que manifestaron haber tenido alguna experiencia con alguna técnica de relajación, meditación, yoga, taichi, etc. De los 50 sujetos que pasaron a formar parte del estudio, 25 formaron el grupo control y los otros 25 el grupo experimental. La asignación a uno u otro grupo se realizó aleatoriamente controlándose la variable sexo para que hubiese un número similar de chicos y chicas en ambos grupos y así evitar la posible interferencia de dicha variable en los resultados del estudio.

Una vez formados los grupos, se procedió a la evaluación pre-test de los niveles de motivación de logro y de motivación para el aprendizaje de todos los sujetos participantes en la investigación, mediante la aplicación de los cuestionarios descritos en el apartado Instrumentos.

Posteriormente, se procedió a la aplicación en el grupo experimental del programa de mindfulness Meditación-Fluir (Franco, 2009b). El programa de intervención aplicado tuvo una duración de 10 semanas en sesiones de 1 hora de duración. Adicionalmente, el programa de intervención incluía la práctica diaria de esta técnica durante 30 minutos.

Meditación-Fluir consiste en la repetición de una palabra con una actitud mental libre y abierta, mientras se dirige la atención a la zona del abdomen para notar como entra y sale el aire de la respiración, pero sin tratar de modificar o alterar dicho proceso respiratorio, ya que tan sólo hay que ser conscientes de cómo ocurre dicho proceso de forma natural y sin esfuerzo. La palabra carece absolutamente de significado y está libre de cualquier 
tipo de connotación. Este programa de entrenamiento es completado con diversas metáforas y ejercicios donde se comprende de forma experiencial que los pensamientos y emociones surgen y desaparecen continuamente y que están sometidos a un continuo flujo, aprendiendo, de esta forma, a estar presentes, abiertos y equilibrados frente a cualquier fenómeno o proceso mental o emocional que pueda acontecer. El programa de intervención también implicaba el aprendizaje y práctica de la técnica de mindfulness denominada exploración corporal o body-scan (Kabat-Zinn, 2003).

Meditación-Fluir se encuadra dentro de las técnicas de mindfulness de Segunda Generación (SG-MBI; Van Gordon, Shonin, \& Griffiths, 2015) y ha sido utilizada con éxito en diferentes estudios dentro y fuera del ámbito educativo y con diversas poblaciones, incluidos estudiantes con conductas impulsivas y agresivas, así como pacientes con fibromialgia y mujeres mastectomizadas (Amutio et al., 2018; Franco, Amutio, López-González, Oriol, \& Martínez-Taboada, 2016; Franco, Amutio, Mañas, Sánchez, \& Mateos, 2020).

Al finalizar el programa psicoeducativo de mindfulness Meditación-Fluir en el grupo experimental, se procedieron a evaluar nuevamente los niveles de motivación de logro y de motivación para el aprendizaje de los sujetos de los grupos control y experimental (evaluación post-test), en las mismas condiciones y con los mismos instrumentos que los empleados antes de la intervención. Una vez finalizada la investigación, se impartió el programa de mindfulness a los sujetos del grupo control.

Todos los participantes proporcionaron el consentimiento informado y el estudio fue aprobado por el Comité de Bioética de la Universidad de Almería en España. Los instrumentos y datos fueron codificados de forma alfanumérica, asegurándose la confidencialidad y anonimato, cumpliéndose así con la Ley de Protección de Datos del Comité de Ética para la investigación con Sujetos Humanos (CEISH). Se aplicaron las Directrices internacionales para estudios con sujetos humanos descritos en el Código de Nuremberg y en la Declaración de Helsinki.

\section{Análisis de datos}

Los análisis estadísticos se realizaron con el programa estadístico SPSS versión 22. Se verificó la distribución normal de los datos y los valores atípicos utilizando diagramas de cajas. No se eliminaron datos. Se realizó un análisis de varianza (ANOVA) modelo mixto con 2 medidas repetidas en el factor tiempo (pre-test y post-test) y factor de aleatorización de los grupos, 2 grupos (experimental y control) para examinar el cambio diferencial en la motivación del logro y las dimensiones de la motivación para el aprendizaje. El tamaño del efecto fue indicado por eta cuadrado parcial $\left(\eta^{2} \mathrm{p}\right)$, utilizando los valores límites de referencia que propone Richardson (2011): .0099, .0588 y .1379, indicando tamaño del efecto pequeño, mediano y grande respectivamente.

\section{Resultados}

\section{Motivación del logro}

Se realizó una ANOVA modelo mixto de 2 factores para la motivación del logro, una con medidas repetidas para el factor tiempo y otra para el factor aleatorización de los grupos, es decir, 2 (tiempo -Pre, Post-) x 2 (aleatorización -grupo Experimental, grupo Control). Los resultados indicaron un efecto principal del tiempo $\left(\mathrm{F}=8.022 ; \mathrm{p}=.007 ; \eta^{2} \mathrm{p}=.143\right)$, no hay efecto de grupo $\left(\mathrm{F}=.135 ; p=.715 ; \eta^{2} \mathrm{p}=.003\right)$, y sí se produce efecto para la interacción tiempo-grupo $\left(\mathrm{F}=4.011 ; p=.051 ; \eta_{\mathrm{p}}^{2}=.077\right)$. Como la significatividad estadística es marginal $(p=.051)$, se procedió a realizar las pruebas $t$ de seguimiento intra-grupo. El resultado de esta prueba determinó incrementos entre pre-test y post-test en el grupo experimental $(p=.003)$. No se producen cambios para el grupo control $(p=.130)$.

\section{Motivación para el aprendizaje}

Se realizó ANOVA modelo mixto de 2 factores uno con medidas repetidas 2 (tiempo - pre, post-) x 2 aleatorización (grupo Experimental, grupo Control) para cada dimensión de la motivación para el aprendizaje (Tabla 1). 
En la dimensión motivación intrínseca no hubo efecto del factor tiempo $\left(\mathrm{F}=.006 ; \mathrm{p}=.936\right.$ y $\left.\eta_{\mathrm{p}}^{2}=.000\right)$, ni del grupo $\left(\mathrm{F}=.832 ; p=.36 ; \eta_{\mathrm{p}}^{2}=.017\right)$ y tampoco de la interacción de los factores tiempo- grupo $(\mathrm{F}=.522$; $\left.p=.473 ; \eta_{\mathrm{p}}^{2}=.011\right)$.

Para la dimensión motivación extrínseca no hubo efecto del factor tiempo $\left(\mathrm{F}=.003 ; p=.960 ; \eta^{2} \mathrm{p}=.000\right)$, ni del factor grupo $\left(\mathrm{F}=.019 ; p=.891 ; \eta_{\mathrm{p}}^{2}=.000\right)$ y tampoco de la interacción tiempo-grupo $\left(\mathrm{F}=.125 ; p=.772 ; \eta_{\mathrm{p}}^{2}=.003\right)$.

En relación a la dimensión valoración no hubo efecto determinado por el tiempo $\left(\mathrm{F}=.498 ; \mathrm{p}=.498 ; \eta^{2} \mathrm{p}=\right.$ $.010)$, ni por el grupo $\left(\mathrm{F}=1.046 ; p=.312 ; \eta^{2} \mathrm{p}=.021\right)$ y tampoco por la interacción tiempo - grupo $(\mathrm{F}=2.908$; $\left.p=.095 ; \eta_{\mathrm{p}}^{2}=.057\right)$.

Para la dimensión autoeficacia los resultados mostraron efecto del tiempo $\left(F=14.03 ; p=.000 ; \eta_{p}^{2}=.226\right)$, no mostraron efecto de grupo $\left(\mathrm{F}=2,24 ; p=.141 ; \eta_{\mathrm{p}}{ }_{\mathrm{p}}=.045\right)$ y si fue significativa la interacción tiempo-grupo $\left(\mathrm{F}=10,49 ; p=.002 ; \eta_{\mathrm{p}}^{2}=.179\right)$. La comparación del grupo experimental y control en el post-test muestra mejores resultados para el grupo experimental que para el control $\left(\mathrm{F}=4.424 ; p=.041 ; \eta_{\mathrm{p}}^{2}=.084\right)$. El programa de mindfulness llevado a cabo produce cambios en la dimensión autoeficacia entre el pre-test y post-test del grupo experimental, siendo el tamaño del efecto grande $\left(\eta_{p}^{2}=.337\right)$.

En la dimensión control los resultados mostraron efecto del tiempo $\left(\mathrm{F}=6.37 ; \mathrm{p}=.015\right.$ y $\left.\eta_{\mathrm{p}}^{2}=.117\right)$, no mostraron efecto de grupo $\left(\mathrm{F}=2.86 ; p=.097 ; \eta_{\mathrm{p}}^{2}=.056\right)$ y tampoco efecto de la interacción tiempo-grupo $(\mathrm{F}=2.24$; $\left.p=.141 ; \eta_{\mathrm{p}}^{2}=.045\right)$. En la variable control el programa produce cambios significativos en el grupo experimental, siendo el tamaño del efecto grande $\left(\eta_{\mathrm{p}}^{2}=.144\right)$.

En relación a la dimensión ansiedad, los resultados mostraron un efecto principal del tiempo $(\mathrm{F}=32,09 ; p=$ $\left..000 ; \eta_{\mathrm{p}}^{2}=.401\right)$, no hubo efecto de grupo $\left(\mathrm{F}=.216 ; \mathrm{p}=.644 ; \eta_{\mathrm{p}}^{2}=.004\right)$, pero si hubo efecto de la interacción tiempo-grupo $\left(\mathrm{F}=58,85 ; p=.000 ; \eta_{\mathrm{p}}^{2}=.551\right)$. La comparación del grupo experimental y control en el postest muestra diferencias estadísticamente significativas y con gran tamaño del efecto $\left(\mathrm{F}=6.221 ; p=.016, \eta_{\mathrm{p}}^{2}=.115\right)$. El programa de mindfulness Meditación-Fluir disminuye la ansiedad del grupo experimental de forma significativa, siendo el tamaño del efecto muy grande $\left(\eta_{p}^{2}=.649\right)$.

Tabla 1. ANOVAS de medidas repetidas para las variables en estudio en los grupos experimental y control

\begin{tabular}{|c|c|c|c|c|c|}
\hline & Grupo & $\begin{array}{l}\text { Media (DE) } \\
\text { Pre }\end{array}$ & $\begin{array}{l}\text { Media (DE) } \\
\text { Post }\end{array}$ & Tamaño efecto & \\
\hline \multirow{2}{*}{$\begin{array}{l}\text { Motivación } \\
\text { del logro }\end{array}$} & Experimental & $86.44(26.12)$ & $89.20(25.42)$ & $\mathrm{F}=4.011$ & $\eta_{p}^{2}=.077$ \\
\hline & Control & $83.92(23.7)$ & $86.68(22.68)$ & $\mathrm{F}=4.011$ & $\eta_{p}^{2}=.077$ \\
\hline \multirow{2}{*}{$\begin{array}{l}\text { Motivación } \\
\text { intrínseca }\end{array}$} & Experimental & 20.68 (5.69) & $20.36(5.78)$ & $\mathrm{F}=.206$ & $\eta_{p}^{2}=.004$ \\
\hline & Control & $19(5.66)$ & $19.40(4.38)$ & $\mathrm{F}=.322$ & $\eta_{p}^{2}=.086$ \\
\hline \multirow{2}{*}{$\begin{array}{l}\text { Motivación } \\
\text { extrínseca }\end{array}$} & Experimental & $14.16(3.70)$ & $14(2.44)$ & $\mathrm{F}=.082$ & $\eta_{p}^{2}=.002$ \\
\hline & Control & $13.92(3.10)$ & $14.04(2.18)$ & $\mathrm{F}=.046$ & $\eta_{p}^{2}=.001$ \\
\hline \multirow[t]{2}{*}{ Valoración } & Experimental & $16.44(4.5)$ & $17.28(5.05)$ & $\mathrm{F}=2.850$ & $\eta_{p}^{2}=.056$ \\
\hline & Control & $15.84(4.23)$ & $15.48(3.35)$ & $\mathrm{F}=.523$ & $\eta_{p}^{2}=.011$ \\
\hline \multirow[t]{2}{*}{ Autoeficacia } & Experimental & $21.40(6.86)$ & $24.16(6.96)$ & $\mathrm{F}=24.40 * * *$ & $\eta_{p}^{2}=.337$ \\
\hline & Control & $19.84(6.65)$ & $20.04(6.88)$ & $\mathrm{F}=.128$ & $\eta_{p}^{2}=.003$ \\
\hline \multirow[t]{2}{*}{ Control } & Experimental & $14.04(2.97)$ & $16.08(2.04)$ & $\mathrm{F}=8.09 * *$ & $\eta_{p}^{2}=.144$ \\
\hline & Control & $13.72(2.82)$ & $14,24(3.48)$ & $\mathrm{F}=.526$ & $\eta_{p}^{2}=.011$ \\
\hline \multirow[t]{2}{*}{ Ansiedad } & Experimental & $20.64(5.70)$ & $16.12(4.45)$ & $\mathrm{F}=88.93 * * *$ & $\eta_{p}^{2}=.649$ \\
\hline & Control & $18.68(5.07)$ & $19.36(4.72)$ & $\mathrm{F}=2.013$ & $\eta_{p}^{2}=.040$ \\
\hline
\end{tabular}

Nota. ${ }^{*} \mathrm{p} \leq .051 ; * * \mathrm{p} \leq .01 ; * * * \mathrm{p} \leq .000$ 


\section{Discusión}

Se confirman en parte las hipótesis de partida que se plantearon en el marco teórico, pues el programa de intervención en mindfulness Meditación-Fluir produjo diferencias estadísticamente significativas entre el grupo control y el experimental en la dimensión de motivación de logro y en algunas de las variables de motivación para el aprendizaje (autoeficacia, control y ansiedad), al comparar las puntuaciones medias post-test de ambos grupos. Antes de iniciar el entrenamiento en mindfulness no se encontraron diferencias estadísticamente significativas de partida entre el grupo control y experimental en dichas variables. Así mismo, no se obtuvo ninguna diferencia significativa al comparar las puntuaciones medias pre-test/post-test en el grupo control en ninguna de las dimensiones.

Por lo tanto, a la luz de estos resultados, puede afirmarse que la intervención en mindfulness MeditaciónFluir resulta útil y eficaz a la hora de realizar una intervención psicoeducativa dirigida a la mejora de los niveles de motivación de logro y de motivación para el aprendizaje en estudiantes inmigrantes de origen latinoamericano de primer curso de Bachillerato. Los resultados de este estudio son coherentes con los hallados en otras investigaciones similares que también demostraron efectos positivos de la práctica del mindfulness sobre el estado emocional y motivacional en estudiantes (Amutio et al., 2015; De la Fuente et al., 2010; Evans et al., 2009; Feltman et al., 2009; Ortner et al., 2007; Soriano \& Franco, 2010).

En cuanto a los procesos o mecanismos de acción responsables de la eficacia del programa Meditación-Fluir en los procesos de regulación emocional destaca la exposición a las propias experiencias (incluso a aquellas que son desagradables), ya que genera en las personas una mayor capacidad para tolerar una amplia gama de pensamientos y emociones. Otros procesos psicológicos implicados en la regulación emocional a través del mindfulness son los procesos neurocognitivos y atencionales (Chambers, Gullone, \& Allen, 2009; Simón, 2006). Por último, se han descrito cómo las mejoras en el control ejecutivo producidas por el mindfulness, incrementan y perfeccionan los procesos de regulación emocional (Teper et al., 2014). Más específicamente, el modelo de Teper, Segal, y Inzlicht (2014) propone que través de la consciencia y la aceptación, mecanismos que se relacionan recíproca e interdependientemente, se fomenta el control ejecutivo, que a su vez favorece la regulación emocional. Así, la consciencia de la estimulación (percatarse de la presencia de una emoción como la ira o el miedo) junto con la aceptación de la emoción (observarla sin pretender controlarla adoptando una postura imparcial o ecuánime) se traduce en un control ejecutivo que favorece la regulación de las emociones, pudiendo las personas responder de forma más adaptativa.

El programa no produjo cambios en tres de las seis dimensiones de la variable motivación para el aprendizaje (motivación intrínseca, motivación extrínseca y valoración). Para explicar estos resultados se puede hipotetizar que mindfulness puede afectar de forma más rápida a determinadas dimensiones porque incide de forma diferente en unas que en otras. Por ejemplo, el estudio de De la Fuente et al. (2010) mostró que mindfulness produjo cambios en la variable cansancio emocional y en la mayoría de las dimensiones de las variables de burnout y engagement en una muestra de estudiantes universitarios, pero no se obtuvieron cambios en las dimensiones vigor y dedicación de esta última variable. De forma similar, Kang, Choi, y Ryu (2009) examinaron la eficacia de un programa de mindfulness sobre el estrés, la ansiedad y la depresión en un grupo de estudiantes de enfermería empleando un grupo experimental y un grupo control. Los resultados mostraron que el grupo experimental, en comparación con el grupo control, mejoró de forma significativa en las variables estrés y ansiedad, pero no lo hizo en la variable depresión. En contraposición, el estudio de Oblitas et al. (2019) no encontró ninguna mejoría significativa en la variable estrés en una muestra de estudiantes universitarios. En la misma línea, el estudio de Bergen-Cico, Possemato, y Cheon (2013) encontró una mejoría significativa del bienestar psicológico en una muestra de estudiantes de pregrado después de una intervención de 5 semanas, pero no en ansiedad. Por todo ello, se requieren más investigaciones con programas de entrenamiento suficientemente validados, con grupos aleatorizados, y con seguimiento a largo plazo, que confirmen los resultados obtenidos en esta y otras investigaciones.

Con la práctica de las técnicas de mindfulness, comprendemos que los pensamientos surgen y desaparecen continuamente y que están sometidos a un continuo flujo. Por lo tanto, mediante esta práctica hemos logrado que los estudiantes del estudio aprendan a estar presentes, abiertos y equilibrados frente a cualquier fenómeno o proceso mental o emocional que pueda acontecer. Así, por medio de la práctica las personas llegamos a conocernos mejor, aceptándonos con nuestras fortalezas y limitaciones, sin tener la necesidad de cambiar o 
modificar nada para sentirnos bien con nosotros mismos. Este autoconocimiento y autoaceptación incrementa las competencias emocionales y la autoestima, algo fundamental si la persona en cuestión, además, tiene la condición de inmigrante (Martínez-Taboada et al., 2017; Soriano \& Franco, 2010).

En la misma línea, los resultados obtenidos en la dimensión ansiedad de nuestro estudio coincide con los trabajos de Fernández-Berrocal y Extremera (2005), en los que se concluye que aquellas personas que muestran una gran capacidad para clarificar y regular sus estados emocionales muestran menos sintomatología ansiosa y una mayor satisfacción vital, lo cual se relaciona con un ajuste psicosocial positivo en la adolescencia y una mayor motivación hacia el aprendizaje (López-Cassá, Pérez-Escoda, \& Alegre, 2018; Miranda, Oriol, Amutio, \& Ortúzar, 2019; Lázaro-Visa, Palomera, Briones, Fernández-Fuertes, \& Fernández-Rouco, 2019).

Consideramos que el sistema educativo debe proporcionar a los adolescentes inmigrantes una serie de recursos que les ayuden a enfrentarse con mayor eficacia a las diversas situaciones de estrés que deben afrontar durante dicho período evolutivo, dotándoles de estrategias que les capaciten para un mejor conocimiento de sí mismos y para poder desenvolverse de forma eficaz en un mundo cada vez más flexible y cambiante (LópezGonzález, Amutio, \& Herrero, 2018; Soriano \& Franco, 2010). Las técnicas de mindfulness poseen el aval científico necesario para que el sistema educativo comience a plantearse seriamente la inclusión de dichas técnicas como un recurso disponible al alcance del alumnado para mejorar tanto su desempeño personal y emocional, como cognitivo y académico. Estas técnicas pueden incorporarse de forma transversal dentro de otras disciplinas como la educación física, las artes marciales, la música, la danza, y a las actividades comunes de la vida diaria (Cheng, 2016; Pizarro, Basabe, Amutio, Telletxea, Harizmendi, \& Van Gordon, 2019; Schure, Christopher, \& Christopher, 2008).

Entre las fortalezas de este estudio destaca el hecho de que hay pocas investigaciones sobre la aplicación de programas de mindfulness a la población inmigrante (Franco, Soriano \& Justo, 2010), así como el hecho de que se trata de un estudio aleatorizado. Sin embargo, es evidente, que los datos del presente estudio, aunque positivos, hay que analizarlos con la debida cautela, ya que la muestra de la investigación fue relativamente baja, y se trata de una muestra de voluntarios. Por último, habría que confirmar en investigaciones longitudinales si las mejoras obtenidas en el grupo experimental mediante la participación en el programa Meditación-Fluir, se mantienen a lo largo del tiempo mediante la realización de diversas medidas de seguimiento.

\section{Referencias}

Amutio, A., Franco, C., Sánchez, L.C., Pérez-Fuentes, M.C., Gázquez-Linares, J.J., Van Gordon, W., \& Molero-Jurado, M.M. (2018). Effects of Mindfulness training on sleep problems in patients with fybromialgia. Frontiers in Psychology, 9, 1365, 1-8. doi: 10.3389/ fpsyg.2018.01365

Amutio, A., Franco, C., Gázquez, J. J., \& Mañas, I. (2015). Aprendizaje y práctica de la conciencia plena (Mindfulness) en estudiantes de Bachillerato para potenciar la relajación y la autoeficacia en el rendimiento escolar. Universitas Psicológica, 14, 15-25. doi:10.11144/ Javeriana.upsy14-2.apcp

Arango, J. (2004). Immigration in Spain at the beginning of the XXI century. En Maldonado, J. (edt). Report on the demographic situation in Spain (pp. 161-186). España: Fernando Abril Martorell.

Barca-Lozano, A., Almeida, L. S., Porto-Rioboo, A.M., Peralbo-Uzquiano, M. \& Brenlla-Blanco, J.C. (2012). Motivación escolar y rendimiento: Impacto de metas académicas, de estrategias de aprendizaje y autoeficacia. Anales de Psicología, $28,848-859$. doi:10.6018/analesps.28.3.156101

Bergen-Cico, D., Possemato, K., \& Cheon, S. (2013). Examining the efficacy of a brief mindfulness-based stress reduction (Brief MBSR) program on psychological health. Journal of American College Health, 61, 348-360. doi: 10.1080/07448481.2013.813853

Blanco-Donoso, L.M., García-Rubio, C., Moreno-Jiménez, B., De la Pinta, M. L. R., Moraleda-Aldea, S., \& Garrosa, E. (2017). Intervención breve basada en ACT y mindfulness: estudio piloto con profesionales de enfermería en UCI y Urgencias. International Journal of Psychology and Psychological Therapy, 17, 57-73.

Boekaerts, M., \& Pekrun, R. (2015). Emotions and Emotion Regulation in Academic Settings. Handbook of Educational Psychology (pp 90-104). UK: Routledge Handbooks. doi: 10.4324/9781315688244.ch6

Caqueo-Urízar, A., Flores, J., Irarrázaval, M., Loo, N., Páez, J., \& Sepúlveda, G. (2019). Discriminación percibida en escolares migrantes en el Norte de Chile. Terapia Psicológica, 37, 97-103.

Carbonero, I. (1999). Ansiedad y rendimiento académico. Punto y Aparte, 7, 123-136. 
Chambers, R., Gullone, E., \& Allen, N.B. (2009). Mindful emotion regulation: An integrative review. Clinical Psychology Review, 29, 560-572. doi:10.1016/j.cpr.2009.06.005

Cheng, F. K. (2016). Is meditation conducive to mental well-being for adolescents? An integrative review for mental health nursing. International Journal of Africa Nursing Sciences, 4, 7-19. doi: 10.1016/j.ijans.2016.01.001

Corno, L., \& Snow, R.E. (1986). Adapting teaching to individual differences among learners. En Wittrock, C.M. (edt.). Handbook of research on teaching (pp. 605-629). Londres: MacMillan.

Covington, M.V. (2000). Goal theory, motivation and school achievement: An integrative review. Annual Review of Psychology, 51, 171-200. doi: 10.1146/annurev.psych.51.1.171

De la Fuente, J., Franco, C., \& Mañas, I. (2010). Efectos de un programa de entrenamiento en conciencia plena (mindfulness) en el estado emocional de estudiantes universitarios. Estudios Sobre Educación, 19, 31-52.

Duckworth, A. L., Peterson, C., Matthews, M. D., \& Kelly, D. R. (2007). Grit: perseverance and passion for long-term goals. Journal of Personality and Social Psychology, 92, 1087-1101. doi: 10.1037/0022-3514.92.6.1087

Dunning, D. L., Griffiths, K., Kuyken, W., Crane, C., Foulkes, L., Parker, J, \& Dalgleish, T. (2019). Research review: The effects of mindfulness-based Interventions on cognition and mental health in children and adolescents: A meta-analysis of randomized controlled trials. The Journal of Child Psychology and Psychiatry, 60, 244-258. doi:10.1111/jcpp.12980

Etxebarria, I., Ortiz, M. J., Apodaca, P., Pascual, A., \& Conejero, S. (2015). Pride as moral motive: moral pride and prosocial behaviour/El orgullo como motivación moral: orgullo moral y conducta prosocial. Infancia y Aprendizaje, 38, 746-774. doi: 10.1080/02103702.2015.1076267

Evans, D.R., Baer, R.A. \& Segerstrom, S.C. (2009). The effects of mindfulness and self-consciousness on persistence. Personality and Individual Differences, 47, 379-382. doi:10.1016/j.paid.2009.03.026

Feltman, R., Robinson, M. D., \& Ode, S. (2009). Mindfulness as a moderator of neuroticism-outcome relations: A self-regulation perspective. Journal of Research in Personality, 43, 953-961. doi:10.1016/j.jrp.2009.08.009

Fernández-Berrocal, P., \& Extremera, N. (2005). Inteligencia emocional percibida y diferencias individuales en el meta-conocimiento de los estados emocionales: una revisión de los estudios con el TMMS. Ansiedad y Estrés, 11, 101-122.

Franco, C., Amutio, A., Mañas, I., Sánchez, L.C., \& Mateos, E. (2020). Improving psychosocial functioning in mastectomized women through a mindfulness-based program. Flow meditation. International Journal of Stress Management, 27(1), 74-81. doi: 10.1037/ str0000120

Franco C., Amutio A., López-González, L., Oriol, X., \& Martínez-Taboada, C. (2016). Effect of a Mindfulness Training Program on the Impulsivity and Aggression Levels of Adolescents with Behavioral Problems in the Classroom. Frontiers in Psychology, 7,1385, 1-8. doi: 10.3389/fpsyg.2016.01385

Franco, M. D. G., Beja, M. J., Candeias, A., \& Santos, N. (2017). Emotion Understanding, Social Competence and School Achievement in Children from Primary School in Portugal. Frontiers in Psychology, 8, 1376, 1-15. doi: 10.3389/fpsyg.2017.01376

Franco, C., Soriano, E., \& Justo, E. (2010). Incidencia de un programa psicoeducativo de mindfulness (conciencia plena) sobre el autoconcepto y el rendimiento académico de estudiantes inmigrantes sudamericanos residentes en España. Revista Iberoamericana de Educación, 53, 1-13.

Franco, C. (2009a). Reducción de la percepción del estrés en estudiantes de Magisterio meditante la práctica de la Meditación Fluir. Apuntes de Psicología, 27, 99-109.

Franco, C. (2009b). Meditación Fluir para serenar el cuerpo y la mente. Madrid: Bubok.

Goldberg, S. B., Tucker, R. P., Greene, P. A., Davidson, R. J., Wampold, B. E., Kearney, D. J., \& Simpson, T. L. (2018). Mindfulness-Based interventions for psychiatric disorders: A systematic review and meta-analysis. Clinical Psychology Review, 59, 52-60. doi: 10.1016/j. cpr.2017.10.011

González-Pienda, J. A., Núñez, J. C., González-Pumariega, S., \& García, M. (1997). Autoconcepto, autoestima y aprendizaje escolar [Self-concept, self-esteem and school learning]. Psicothema, 9, 271-289.

Gutiérrez, M., \& López, E. (2012). Motivación, comportamiento de los alumnos y rendimiento académico. Infancia y Aprendizaje, 35, 61-72. doi: 10.1174/021037012798977421

Gutiérrez-Carmona, A., \& Urzúa, A. (2019). ¿Los valores culturales afectan el bienestar humano? Evidencias desde los reportes de investigación. Universitas Psychologica, 18, 1-12. doi: 10.11144/Javeriana.upsy18-1.vcab

Kabat-Zinn, J. (2003). Vivir con plenitud las crisis. Cómo utilizar la sabiduría del cuerpo y la mente para afrontar el estrés, el dolor y la enfermedad. Barcelona: Kairós.

Kang, Y. S., Choi, S. Y., \& Ryu (2009). The effectiveness of a stress coping program based on mindfulness meditation on the stress, anxiety, and depression experienced by nursing students in Korea. Nurse Education Today, 29, 538-543. doi: 10.1016/j.nedt.2008.12.003 
Karimi, S., \& Sotoodeh, B. (2019). The mediating role of intrinsic motivation in the relationship between basic psychological needs satisfaction and academic engagement in agriculture students. Teaching in Higher Education, 1-17. doi: 10.1080/13562517.2019.1623775

Klingbeil, D. A., \& Renshaw, T. L. (2018). Mindfulness-based interventions for teachers: A meta-analysis of the emerging evidence base. School Psychology Quarterly, 33, 501-511. doi: 10.1037/spq0000291

Krick, A., \& Felfe, J. (2019). Who benefits from mindfulness? The moderating role of personality and social norms for the effectiveness on psychological and physiological outcomes among police officers. Journal of Occupational Health Psychology (online). doi: 10.1037/ocp0000159

Lam K.K.L., \& Zhou M. (2019). Examining the relationship between grit and academic achievement within K-12 and higher education: A systematic review. Psychology in the Schools, 56, 1654-1686. doi:10.1002/pits.22302

Langer, A. I., Ulloa, V. G., Cangas, A. J., Rojas, G., \& Krause, M. (2015). Mindfulness-based interventions in secondary education: a qualitative systematic review/Intervenciones basadas en mindfulness en educación secundaria: una revisión sistemática cualitativa. Estudios de Psicología, 36, 533-570. doi: 10.1080/02109395.2015.1078553

Langer, E. J. (2000). The construct of mindfulness. Journal of Social Issues, 56, 1-9. doi:10.1111/0022-4537.00148

Lázaro-Visa, S., Palomera R., Briones E., Fernández-Fuertes A.A., \& Fernández-Rouco, N. (2019). Bullied Adolescent's Life Satisfaction: Personal Competencies and School Climate as Protective Factors. Frontiers in Psychology, 10, 1691, 1-11.doi: 10.3389/fpsyg.2019.01691

León, B. (2008). Atención plena y rendimiento académico en estudiantes de educación secundaria. European Journal of Education and Psychology, 1, 17-26.

López-Cassá, E., Pérez-Escoda, N., \& Alegre, A. (2018). Competencia emocional, satisfacción en contextos específicos y satisfacción con la vida en la adolescencia. Revista de Investigación Educativa, 36, 57-73. doi: 10.6018/rie.36.1.273131

López-González, L., Amutio, A., \& Herrero, D. (2018). The Relaxation-Mindfulness Competence of Secondary and High School students and its influence on classroom climate and academic performance. European Journal of Education and Psychology, 11, 5-17.

López-González, L., Amutio, A., Oriol, X., \& Bisquerra, R. (2016). Hábitos relacionados con la relajación y la atención plena (mindfulness) de estudiantes de secundaria: influencia en el clima de aula y el rendimiento académico [Habits Related to Relaxation and Mindfulness of High School Students: Influence on Classroom Climate and Academic Performance]. Revista de Psicodidáctica/ Journal of Psychodidactics, 21, 121-138. doi: 10.1387/RevPsicodidact.13866

Manassero, M., \& Vázquez, A. (1998). Validación de una escala de motivación de logro. Psicothema, 10, 333-351.

Maquilón, J., \& Hernández, F. (2011). Influencia de la motivación en el rendimiento académico de los estudiantes de Formación profesional. Revista Electrónica Interuniversitaria de Formación del profesorado, 14, 81-100.

Martínez-Taboada, C., Mera M. J., Amutio, A., Castañeda, X., Felt, E., \& Nicolae, G. (2017). The Impact of Cultural Dissonance and Acculturation Orientations on Immigrant Students' academic performance. Universitas Psicológica, 16, 1-14. doi: 10.11144/Javeriana.upsy16-5.icda

Miranda, R., Oriol, X., Amutio, A., \& Ortúzar, H. (2019). Bullying en la adolescencia y satisfacción con la vida: ¿puede el apoyo de los adultos de la familia y de la escuela mitigar este efecto? [Adolescent Bullying Victimization and Life Satisfaction: Can Family and School Adult Support Figures Mitigate this Effect?] Revista de Psicodidáctica/Journal of Psychodidactics, 24, 39-45. doi: 10.1016/j. psicod.2018.07.001

Ministerio de Educación y Formación Profesional (2019). Estadística de las Enseñanzas no universitarias. Curso escolar $2017 / 2018$. Subdirección General de Estadística y Estudios del Ministerio de Educación y Formación Profesional. Recuperado de http://estadisticas.mecd.gob.es/EducaJaxiPx/Tabla.htm?path=/Educacion/Alumnado/Matriculado/2017-2018RD/Extranjeros//10/\&file=Extran3. px\&type $=$ pcaxis $\& \mathrm{~L}=0$

Oblitas, L. A., Soto, D.E., Anicama, J. C., \& Arana, A. A. (2019). Incidencia del mindfulness en el estrés académico en estudiantes universitarios: Un estudio controlado. Terapia Psicológica, 37, 116-128. doi: 10.4067/S0718-48082019000200116

OECD (2015). ¿Se puede cerrar la brecha de rendimiento entre los alumnos inmigrantes

y no inmigrantes? PISA in Focus, 53. Recuperado desde http://www.mecd.gob.es/dctm/inee/pisa-in-focus/pisa-in-focus-n53esp.pdf?documentId $=0901 \mathrm{e} 72 \mathrm{~b} 81 \mathrm{ebd} 807$

OECD (2018). How do the performance and well-being of students with an immigrant background compare across countries? PISA in Focus, 82. https://doi.org/10.1787/a9e8clab-en

Oriol, X., Miranda, R., Oyanedel, J. C., \& Torres, J. (2017). The role of self-control and grit in domains of school success in students of primary and secondary school. Frontiers in psychology, 8, 1716. doi:10.3389/fpsyg.2017.01716

Oriol, X., Amutio, A., Mendoza, M., Da Costa, S., \& Miranda, R. (2016). Emotional creativity as predictor of intrinsic motivation and academic engagement in university students: the mediating role of positive emotions. Frontiers in Psychology, 7, 1243. doi: 10.3389/ fpsyg.2016.01243 
Ortner, C.N., Kilner, S.J., \& Zelazo, P.D. (2007). Mindfulness meditation and reduced emotional interference on a cognitive task. Motivation and Emotion, 31, 271-283. doi: 10.1007/s11031-007-9076-7

Pintrich, P., Smith, D., Garcia, T., \& McKeachie, W. (1991). A manual for the use of the motivational strategies for learning questionnaire (MSLQ). Universidad de Michigan.

Pizarro, J. J., Basabe, N., Amutio, A., Telletxea, S., Harizmendi, M., \& Van Gordon, W. (2019). The mediating role of shared flow and perceived emotional synchrony on compassion for others in a mindful-dancing program. Mindfulness, 10, 1-15. doi: 10.1007/ s12671-019-01200-Z

Reher, D., M. Requena, M., \& Sanz, A..(2011). ¿España en la encrucijada? Consideraciones del cambio de ciclo migratorio. Revista Internacional de Sociología, 69, 9-44.

Richardson, J.T.E. (2011). Eta squared and partial eta squared as measurements of effect size in educational research. Educational Research Review, 6, 135-147. doi:10.1016/j.edurev.2010.12.001

Roche, A. I., Kroska, E. B., \& Denburg, N. L. (2019). Acceptance and mindfulness-based interventions for health behavior change: Systematic reviews and meta-analyses. Journal of Contextual Behavioral Science, 13, 74-93. doi: 10.1016/j.jcbs.2019.06.002

Rodríguez, S., Núñez, J.C., Valle, A., Freire, C., Ferradas, M.M., \& Rodríguez-Llorente, C. (2019). Relationship Between Students' Prior Academic Achievement and Homework Behavioral Engagement: The Mediating/Moderating Role of Learning Motivation. Frontiers in Psychology, 10, 1047, 1-10. doi: 10.3389/fpsyg.2019.01047

Sapthiang, S., Van Gordon, W., \& Shonin, E. (2019). Mindfulness in schools: a health promotion approach to improving adolescent mental health. International Journal of Mental Health and Addiction, 17, 112-119. doi: 10.1007/s11469-018-0001-y

Schure, M.B., Christopher, J. \& Christopher, S. (2008). Mind-body medicine and the art of self- care: Teaching mindfulness to counseling students through yoga, meditation, and qigong. Journal of Counseling \& Development, 86, 47-56.

Sevilla, J., Santesteban, O., Pryor, I., McGorry, P., \& Álvarez, M. (2018). Web-based mindfulness interventions for mental health treatment: Systematic review and meta-analysis. JMIR Mental Health, 5(3), 1-16. e10278. doi:10.2196/10278

Simón, V. M. (2006). Mindfulness y neurobiología. Revista de Psicoterapia, 17, 5-30.

Solhaug, I., de Vibe, M., Friborg, O., Sørlie, T., Tyssen, R., Bjørndal, A., \& Rosenvinge, J. H. (2019). Long-term Mental Health Effects of Mindfulness Training: a 4-Year Follow-up Study. Mindfulness, 10, 1661-1672. doi: 10.1007/s12671-019-01100-2

Soriano, E., \& Franco, C. (2010). Mejora de la autoestima y de la competencia emocional en adolescentes inmigrantes sudamericanos residentes en España a través de un programa psicoeducativo de Mindfulness (conciencia plena). Revista de Investigación Educativa, $28,297-312$.

Soriano, E. (2005). Diagnóstico de la identidad cultural en las escuelas multiculturales e intervención educativa. En Soriano, E. (edt). La interculturalidad como factor de calidad educativa (pp.175-208). Madrid: La Muralla

Soriano, E. (2012). Immigration to European Nations. En Banks, J. (ed). Encyclopedia of Diversity in Education (1141-1143). C.A: SAGE Publications.

Teper, R., Segal, Z. V., \& Inzlicht, M. (2014). Inside the mindful mind: How mindfulness enhances emotion regulation througfh improvements in executive control. Current Directions in Psychological Science, 23, 302-307. doi: 10.1177/0963721413495869

Urzúa, A., Basabe, N., Pizarro, J. J., \& Ferrer, R. (2017). Afrontamiento del estrés por aculturación: inmigrantes latinos en Chile. Universitas Psychologica, 16, 1-13. doi: doi.org/10.11144/Javeriana.upsy16-5.aeai

Van Gordon, W., Shonin, E., \& Griffiths, M. D. (2015). Towards a second generation of mindfulness-based interventions. Australian \& New Zealand Journal of Psychiatry, 49, 591-592. doi:10.1177/0004867415577437

Weinstein, N., Brown, K.W., \& Ryan, R.M. (2009). A mul-method examination of the effects of mindfulness on stress attribution, coping, and emotional well-being. Journal of Research in Personality, 43, 374-385. doi:10.1016/j.jrp.2008.12.008 